\title{
A Study of p53 immunostaining in prostate carcinomas: Correlation with Gleason's score
}

\author{
Roopa Urs A.N ${ }^{1 *}$, Manjunath GV$^{2}$, Suchitha $\mathrm{S}^{2}$ and Hugara Siddalingappa ${ }^{1}$ \\ ${ }^{1}$ Department of Pathology PK Das Institute of Medical Sciences, Vaniamkulam, Palakkad \\ ${ }^{2}$ Department of Pathology JSS Medical College, Mysore, India
}

\begin{abstract}
Background: Prostate cancer is the second most common cause of cancer and the sixth leading cause of cancer death among men worldwide. In view of the above, early diagnosis and effective treatment of the disease are immensely important. The increasing number of options for the treatment of prostate cancer has made the prognostic evaluation of the disease even more important. P53 is a tumor suppressor gene characterised by a highly proliferative pattern and an aggressive behaviour. The Objectives was to study the immunostaining patterns of p53 in prostate cancers and to compare the results with Gleason's score.
\end{abstract}

Methods: Fifty cases of histopathologically proven prostate carcinomas diagnosed on needle biopsies and transurethral resection specimens was studied in JSS medical college and hospital, mysore for a period of 3 years and histopathological grade was assessed using Gleason grading system. Immunohistochemistry (IHC) for p53 was done on paraffin embedded wax sections.

Result: p53 staining was positive in $47(94 \%)$ cases out of 50 cases, three $(6 \%)$ cases were negative. Although there was an increase in positive p53 staining with increased Gleason's score, it was not statistically significant ('p' value $=0.068$ ).

Conclusion: $\mathrm{p} 53$ is a tumor suppressor gene, that express high proliferative pattern. It can be used as a prognostic factor. The immunoreactivity of p53 marker with increased tumor grade can benefit patients with appropriate targeted treatment and increase their survival period.

Keywords: Prostate Cancer, Gleason's Score, p53.

\section{Introduction}

Prostate cancer is the second most common cause of cancer and the sixth leading cause of cancer death among men worldwide. The mortality rates are highest in the Caribbean and lowest in South Central Asia, but this partly reflects varying data quality worldwide. Prostate cancer is strongly related to age with the highest mortality rates being in older males. Rise in the incidence of the prostate cancer has partly attributed with screening of prostate specific antigen (PSA) level. ${ }^{1}$ Prostate cancer is not only significant for its lethality but also for the extremely high morbidity associated with it. ${ }^{2}$

Patients generally do not experience symptoms during early stage and are unlikely to seek medical help until the disease has progressed. Thus, prostate cancer is acknowledged as a major health problem and with the advent of screening tests like digital rectal examination(DRE) and prostate specific antigen test, more patients are being diagnosed in earlier stages. With delay in early diagnosis of the lowgrade tumor, the quality or length of patient's life is not significantly changed, but a high-grade tumor in a young person might spread quickly and lead to the patient's death within two years. ${ }^{3}$
Now-a-days, prostatic needle biopsies and transurethral resected specimens are being increasingly used to diagnose prostatic carcinomas. Histopathologically, once the diagnosis of adenocarcinoma is made, it is graded using Gleason scoring system. Gleason system is one of the best prognostic predictor ${ }^{4}$ recommended by World health organization and very acceptable by majority of urologists and radiotherapists. The Gleason grading system, named after Donald F. Gleason, is a unique histopathological method for grading prostate cancer based solely on the tumor architecture. ${ }^{4,5}$ It is important preoperative predictor of the behavior of prostate cancer and is used to help in making decisions about treatment for localized prostate cancer. ${ }^{6,7}$ It is also used to predict relapse in patients receiving hormone therapy for bone metastasis of prostate cancer. ${ }^{8}$ But recent studies using various biomarkers have proved that many prostatic carcinomas have been over graded or under graded using Gleason scoring system. ${ }^{5}$ This may be attributed to its subjective nature and has aroused the need to search for novel markers which are more objective and can predict the behavior of prostatic carcinoma $^{5}$

P53 originally referred to a 53-Kilodalton Phosphoprotein, the product of a 20 - Kilobase gene on short arm of 
chromosome 17. It plays critical role as a cancer suppressor. p53 is involved in the regulation of cell cycle, causing cell cycle arrest at $\mathrm{G} 1$ phase and in certain cell types precipitating apoptosis. $^{9}$ The importance of p53 in the pathogenesis of prostatic adenocarcinoma was first postulated by Rubin SJ et $\mathrm{al}^{10}$ and Isaacs WB et $\mathrm{al}^{11}$, who demonstrated mutations of p53 gene in prostate cell lines and in primary human prostatic adenocarcinoma. Many studies have suggested a significant association between p53 immunoreactivity and prostate carcinomas characterised by a highly proliferative pattern and an aggressive behaviour. ${ }^{12,13}$ A good correlation has been found between the detection of mutations at the molecular level and the over expression of the protein as detected immunohistochemically. ${ }^{14}$

Despite improvements in early detection of prostate cancer as a result of DRE and PSA screening, we still lack molecular markers to effectively distinguish patients with high risk of disease progression from the indolent majority. Considering the proven correlation between Gleason's grading and prognosis of prostate cancer, p53 study is undertaken to investigate the frequency of expression of the marker in prostate cancer and its probable relation with Gleason's score.

\section{Materials and Methods}

In this study, fifty cases of Prostate carcinomas diagnosed on needle biopsies and transurethral resection specimens were studied in JSS medical college and hospital, Mysore for a period of 3years. Ethical committee clearance was obtained. The patient's medical records were reviewed to obtain patient's clinico-pathological parameters, including age at diagnosis, pretreatment sPSA values, digital rectal examination findings, type of procedure, ultrasonography and CT scan findings. All surgically removed prostate carcinoma tissues was fixed in formalin followed by paraffin embedding and stained with haematoxylin and eosin. Sections were studied and histological diagnosis was given according to WHO classification. All cases were assigned Gleason score by Gleason grading system.

IHC for p53 was done on $4 \mu \mathrm{m}$ thick paraffin embedded wax sections on poly-1 lysine coated slides. Antigen retrieval was done in tri sodium citrate buffer at $\mathrm{pH}$ 6. p53 antibody (Novocastra Code No RTU-p53-DO7) was used for p53 antigen by one step horseradish peroxidase (HRP) polymer method. A section from a poorly differentiated breast carcinoma was taken as positive control whereas sections treated with tris-buffer solution instead of primary antibody was used as negative control. Strong brown nuclear immunoreactivity was considered as positive staining.

The immunoquantification was performed using percentage of tumor cells that react with the antibody. Each slide was evaluated at $\mathrm{x} 40$ magnification in order to find areas with maximum positive cells. Then these areas were examined at $x 400$ magnification and the percentage of positive cells to total cells was calculated. At least 500 cells were counted, and only the cells that were definitely positive was considered.

A semiquantitative scoring system was employed to assess the level of p53 reactivity. Grade 0 was assigned when no staining was observed, grade 1 when $<10 \%$ of tumor cell nuclei were reactive, grade 2 when $>10 \%$ but $<33 \%$ of the nuclei stained, grade 3 for $>33 \%$ of nuclei were positive.

Data was analysed using Epi-info statistical software. Univariate analysis was done and expressed in mean and percentages. Bivariate analysis was done using Spearman's correlation coefficient, to find out the strength of association between histopathological report results and Gleason's score. The association of p53 as a categorical variable was determined by chi square test. Results were interpreted at alpha error level of $5 \%$. p value of $<0.05$ was considered as statistically significant.

\section{Result}

In the present study, the age group of patients ranged from 44 to 86 years, with a mean age of 69.9 years. Patients predominantly presented with acute urinary retention (AUR) \& lower urinary tract symptoms (LUTS). Digital rectal examination was abnormal in $45 \%$ of cases. $60 \%$ patients had grade II prostatomegaly on ultrasonography. All cases displayed features of Acinar Adenocarcinoma (Ordinary type) of which glandular pattern (84\%), followed by cribriform (56\%) were common patterns. Majority $(70 \%)$ had poorly differentiated adenocarcinoma followed by moderately-poorly differentiated (24\%) [Table-1] No cases of well differentiated adenocarcinoma was encountered during the study period. Perineural invasion (PNI) was seen in $48 \%$ of the cases. One case ( $2 \%$ ) showed metastasis to bone which was picked up by Positron Emission Tomography (PET).

With p53 staining, fourth seven (94\%) cases out of 50 cases showed positive staining for $\mathrm{p} 53$, three $(6 \%)$ cases were negative. Based on tumor differentiation $15(30 \%)$ were moderately differentiated and $35(70 \%)$ were poorly differentiated. None of them were well differentiated. Seven (14\%) cases were graded 1 (1-10\%) of which four were moderately differentiated and three were poorly differentiated. 14 (28\%) cases were graded 2 (10-33\%) in which five were moderately differentiated and nine were poorly differentiated and $26(52 \%)$ cases were graded 3 $(>33 \%)$, of which four were moderately differentiated and 22 were poorly differentiated . [Table -1, Figure 1,2,3] 
Table 1: Frequency of p53 in relation to differentiation and Gleason grade.

\begin{tabular}{|c|c|c|c|c|}
\hline \multirow{2}{*}{$\mathrm{p53}$} & \multicolumn{4}{|c|}{ Gleason Grade } \\
\cline { 2 - 5 } & Moderately differentiated & Poorly differentiated & Frequency & Percentage (\%) \\
\hline 0 & 2 & 1 & 3 & 6.0 \\
\hline $1-10 \%$ & 4 & 3 & 7 & 14.0 \\
\hline $10-33 \%$ & 5 & 9 & 26 & 28.0 \\
\hline$>33 \%$ & 4 & 22 & $\mathbf{5 0}$ & 52.0 \\
\hline Total & $\mathbf{1 5}$ & $\mathbf{3 5}$ & $\mathbf{1 0 0 . 0}$ \\
\hline
\end{tabular}

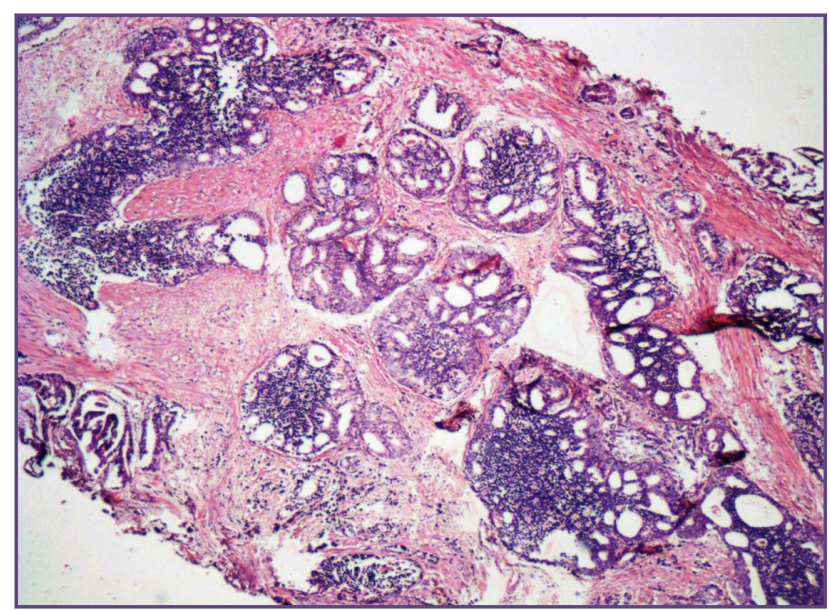

Fig. 1: Photomicrograph showing poorly differentiated adenocarcinoma with tumor cells arranged in cribriform pattern with lymphocytic infiltration. Gleason's score 4+4=8 (H\&E, x100).

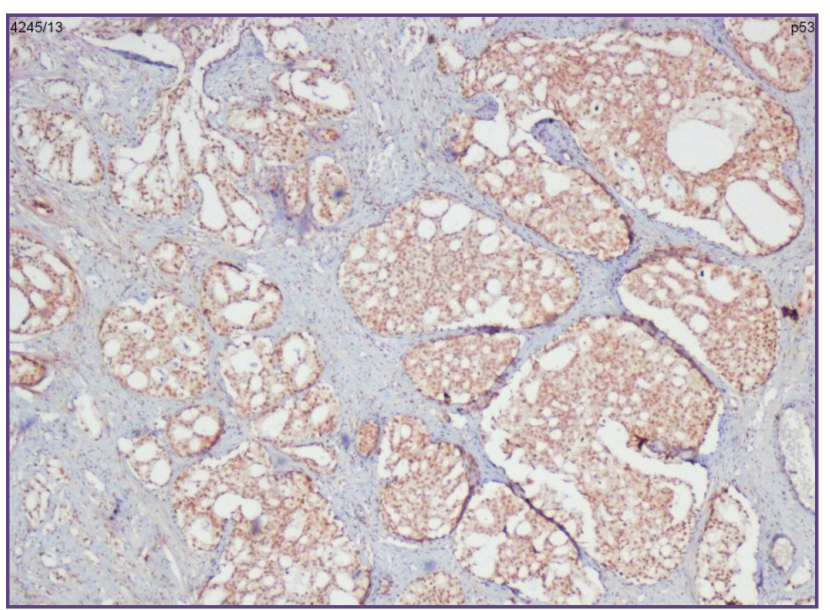

Fig. 2: Photomicrograph showing poorly differentiated adenocarcinoma with $3+$ positivity for p53 immunostain. Gleason's score $4+4=8(p 53, x 100)$.

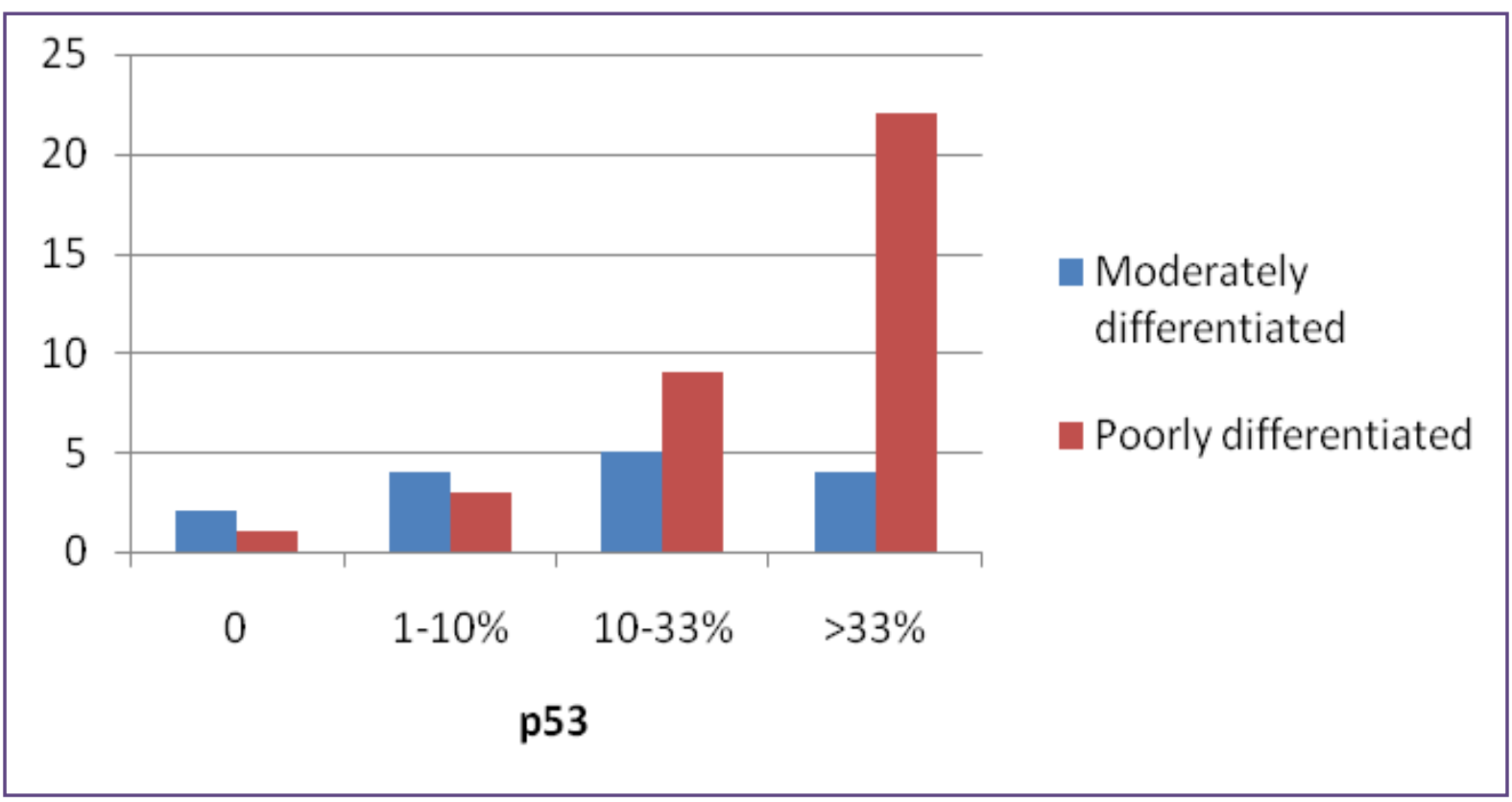

Fig. 3 : Comparison of results of p53 and Gleason's score among study samples. 


\section{Discussion}

In our study, majority (70\%) of tumors were poorly differentiated adenocarcinoma followed by moderately differentiated adenocarcinomas (30\%). Whereas, similar studies done by Madani SH et $\mathrm{al}^{3}(51 \%)$, Shirley SE et $\mathrm{al}^{15}(60 \%)$, Catalona WJ et $\mathrm{al}^{16}(60 \%)$ have also reported majority of prostatic adenocarcinomas are poorly differentiated with Gleason score between 8-10. On the contrary, Chiusa L et $\mathrm{al}^{17}$, Petrescu A et $\mathrm{al}^{9}$ (56.6\%) have reported moderately differentiated adenocarcinoma (Gleason score 5-7) are the predominant type. The possible contributing factors for this differentiation difference might be genetic, environmental, racial, dietary factors or interobserver variability in Gleason scoring or the authors who have still considered Gleason score 7 to be of moderately differentiated grade.

p53 over expression has been investigated independently in a large number of different malignancies for their potential value as a prognostic marker. Mutation of the p53 tumor suppressor gene is a common genetic alteration in malignant human tumors and can be immunohistochemically detected. ${ }^{9}$ In our study, Out of 50 cases, 47(94\%) cases showed strong nuclear positive staining for p53. Only three $(6 \%)$ cases were negative. In the literature, the range of incidence of p53 positive immunoexpression in prostate cancers has been reported ranging from 4 to $61 \% .{ }^{19}$ Most of this variation are attributed to methodological differences in tissue sampling, antibody clone used and scoring.

Although there was an increase in positive p53 staining with increased Gleason's score, it was not statistically significant ('p' value $=0.068$ ) in our study. The results were correlated with studies done by Visakorpi $\mathrm{T}$ et $\mathrm{al}^{12}$, Bookstein R et $\mathrm{al}^{20}$, Shurbaji MS et $\mathrm{al}^{21}$, Grignon $\mathrm{DJ}^{22}$ et al, Cappello $\mathrm{F}$ et al ${ }^{23}$, Petrescu A et al. ${ }^{9}$ According to them, there is significant association between p53 protein over expression and increased Gleason score. These results strongly imply that p53 mutations play a role in the pathogenesis of a subset of prostate cancers. But the precise molecular role played by the over expressed p53 protein in mediating oncogenesis in prostate epithelium remains to be determined. In contrary, study by Lin JT et al ${ }^{13}$, Madani SH et $\mathrm{al}^{3}$, had no statistically significant correlation between p53 positivity and increased Gleason's score and have said formalin fixation reduces expression of p53.

Whereas, Borre et $\mathrm{al}^{24}$ have showed the accumulation of p53 have a special correlation with patients survival. The presence and activity of p53 was greatly associated with the cell proliferation marker (MIB-1) and the level of p53 activity was an important independent prognostic factor that was inversely associated with patient survival. But Sasor et $\mathrm{al}^{25}$, showed that there is no significant difference between the presence of p53 in low and high grade tumors but there is only a positive relationship between the expressions of $\mathrm{Ki}-67$ and p53 in patients with low-grade prostate cancer. Also, he has said that increased histologic grade and presence of metastases suggest that p53 expression may be linked to the tumor behavior.

Bookstein $\mathrm{R}$ et $\mathrm{al}^{20}$, showed abnormal nuclear p53 expression may be an early event in prostatic carcinoma progression, Where as, Schlomm $\mathrm{T}$ et $\mathrm{al}^{18}$ and Visakorpi $\mathrm{T}$ et $\mathrm{al}^{12}$ have reported low frequencies of $\mathrm{p} 53$ positivity by immunohistochemistry and poor prognosis.

\section{Conclusion}

To conclude $\mathrm{p} 53$ is a tumor suppressor gene defines a small subgroup of highly malignant tumors. p53 immunoreactivity in prostate carcinomas are seen with high proliferative pattern and suggest the aggressive behavior of tumour. Also immunoreactivity of p53 marker with increased tumor grade can benefit patients with appropriate targeted treatment and increase their survival time. It can be used as prognostic indicator but further studies are required with more number of cases to determine their biologic role and progression of disease in prostate cancer.

\section{Reference:}

1. Ferlay J, Foucher ES, Tieulent LJ, Rosso S. Cancer incidence and mortality patterns in Europe: Estimates for 40 countries in 2012. European Journal of Cancer 2013;49:1374- 1403.

2. Verma R, Gupta V, Singh J, Verma M, Gupta G et al. Significance of p53 and Ki67 expression in prostate cancer. Urology Annals 2015;7(4):488-493

3. Madani SH, Ameli S, Khazaei S, Kanani M, Izadi B. Frequency of Ki-67 (MIB-1) and P53 expressions among patients with prostate cancer. Indian J Pathol Microbiol 2011;54:688-91

4. Gleason DF, Mellinger GT. Prediction of prognosis for prostatic adenocarcinoma by combined histological grading and clinical staging. J Urol 1974; 111: 58-64.

5. Gleason DF. Classification of prostatic carcinomas. Cancer Chemother Rep. Part 1. 1966; 50(3): 125-8.

6. Mellinger GT, Gleason D, Bailar J. The histology and prognosis of prostatic cancer. J Urol 1967; 97(2):331-7.

7. Poulos CK, Daggy JK, Cheng L. Preoperative prediction of Gleason grade in radical prostatectomy specimens: the influence of different Gleason grades from multiple positive biopsy sites. Mod Pathol 2005; 18: 228-34.

8. Tsukamoto T, Kumamoto Y, Umehara T, Takahashi A, Shimazaki J, Ohshima $\mathrm{H}$ et al. Clinical study of bone-related relapse in prostate carcinoma. Adv Exp Med and Biol 1992; 324: 283-93.

9. Petrescu A, Mârzan L, Codreanu O, Niculescu L. Imunohistochemical detection of $\mathrm{p} 53$ protein as a prognostic 
indicator in prostate carcinoma. Rom morphol Embryol 2006; 47(2):143-6.

10. Rubin SJ, Hallahan DE, Ashman CR, Brachman DG, Beckett MA, Virudachalam Set al. Two prostate carcinoma cell lines demonstrate abnormalities in tumor suppressor genes. J Surg Oncol 1991;46(1):31-6.

11. Isaacs WB, Carter BS, Ewing CM. Wild-type $\mathrm{p} 53$ suppresses growth of human prostate cancer cells containing mutant p53 alleles. Cancer Res 1991;51(17):4716-20.

12. Visakorpi T, Kallioniemi OP, Heikkinen A, Koivula T, and Isola J. Small Subgroup of Aggressive, Highly Proliferative Prostatic Carcinomas Defined by p53 Accumulation. J Natl Cancer Inst 1992; 84(11): 883-887

13. Lin JT, Wang JS, Jiann BP, Yu CC, Tsai JY, Huang JK et al. Correlation of p53 protein accumulation and Bcl-2 overexpression with histopathological features in prostatic cancer. J Formos Med Assoc 2005;104 (11):864-7.

14. Wertz IE, Deitch AD, Gumerlock PH, Gandour-Edwards R, Chi SG, DeVere White RW. Correlation of genetic and immunodetection of TP53 mutations in malignant and benign prostate tissues. Hum Pathol 1996;27(6):573-80.

15. Shirley SE, Escoffery CT, Sargeant LA, Tulloch T. Clinicopathological features of prostate cancer in Jamaican men. BJU International 2002; 89:390-95.

16. Catalona WJ, Antenor JA, Roehl KA, Moul JW. Screening for prostate cancer in high risk populations. J Urol 2002;168:1980-84.

17. Chiusa L, Galliano D, Formiconi A, Di Primio O, Pich A. High and low risk prostate carcinoma determined by histologic grade and proliferative activity. American cancer society $1997 ; 79: 1956-63$
18. Schlomm T, Iwers L, Kirstein P, Jessen B, Ko"llermann $\mathrm{J}$, Minner S, et al. Clinical significance of p53 alterations in surgically treated prostate cancers. Modern Pathology 2008;21: 1371-1378

19. Jemal A, Siegel R, Ward E, Murray T, Xu J, Thun MJ. Cancer statistics, 2007. CA: A Cancer Journal for Clinicians 2007;57(1):43-66.

20. Bookstein R, MacGrogan D, Hilsenbeck SG, Sharkey F, Allred DC. p53 is mutated in a subset of advanced-stage prostate cancers. Cancer Res 1993; 53:3369-73

21. Shurbaji MS, Kalbfleisch JH, Thurmond TS. Immunohistochemical detection of p53 protein as a prognostic indicator in prostate cancer. Hum Pathol 1995;26:106-9.

22. Grignon DJ, Caplan R, Sarkar FH, Lawton CA, Hammond EH, Pilepich MV, et al. p53 Status and Prognosis of Locally Advanced Prostatic Adenocarcinoma: a Study Based on RTOG 8610. J Natl Cancer Inst 1997; 89:158-65

23. Cappello F, Ribbene A, Campanella C, Czarnecka AM, Anzalone R, Bucchieri F, Palma A, Zummo G. The value of Imunohistochemical research on PCNA, p53 and heat shock proteins in prostate cancer management: a review. European Journal of Histochemistry 2006; 50(1):25-34.

24. Borre M, Stausbot-Gron B, Overgaard J. P53 accumulation associated with bcl-2, the proliferation marker MIB-1 and survival in patients with prostate cancer subjected to watchful waiting. J Urol 2000;164:716-21.

25. Sasor A, Wagrowska-Danilewicz M, Danilewicz M. Ki67Antigenand P53 protein expression in benign and malignant prostatic Lesions. Pol J Pathol 2000; 51:31-6.

*Corresponding author:

Dr. Roopa URS A.N, Assistant Professor, Department of Pathology, No.201, A block, 4th main, J.P.Nagar, Mysore-570031 India

Phone: +91 8884141327, 8248124351

Email: roopaurspr@gmail.com

Financial or other Competing Interests: None. 